DOI 10. 18307/2019. 0426

(C) 2019 by Journal of Lake Sciences

\title{
太湖梅梁湾水体及沉积物中微囊藻毒素含量垂向分布特征”
}

\author{
舒秀波 ${ }^{1,2}$, 谢丽强 ${ }^{2}$,万 翔 ${ }^{2,3}$,姚 䂞 ${ }^{2,3}$, 薛庆举 ${ }^{2}$, 李金娟 ${ }^{1 * *}$ \\ ( 1 : 贵州大学资源与环境工程学院, 贵阳 550025) \\ (2: 中国科学院南京地理与湖泊研究所湖泊与环境国家重点实验室,南京 210008) \\ (3: 中国科学院大学,北京 100049)
}

\begin{abstract}
摘 要: 为探究太湖梅梁湾水体及沉积物中微囊藻毒素 (MC-LR、MC-RR、MC-YR) 含量的垂向分布特征, 于 2018 年 5 月 采集梅梁湾 6 个点位表层水、上覆水、混合水、间隙水以及柱状沉积物样品, 并采用超高效液相色谱一串联质谱法分析样 品中微囊藻毒素的含量. 分析结果表明: 水体中 (表层水、上覆水、混合水以及间隙水) MC-LR、MC-RR、MC-YR 的浓度范围 分别为 $11.80 \sim 1297.14 、 2.50 \sim 818.40 、 1.80 \sim 176.00 \mathrm{ng} / \mathrm{L}$, 表层水、上覆水以及混合水中 MC-LR 的浓度高于 MC-RR 和 MCYR, MC-RR 和 MC-YR 之间差别较小,而间隙水中 MCs 三种异构体浓度大小顺序为: MC-LR $>$ MC-RR $>$ MC-YR; 垂向分布 上, 间隙水中 MCs 异构体 (MC-LR、MC-RR、MC-YR) 浓度均远高于表层水、上覆水以及混合水,表层水 MCs 异构体浓度略 高于上覆水, 混合水 MCs 异构体浓度介于表层水和上覆水之间. 对沉积物的研究发现, $1 \sim 10 \mathrm{~cm}$ 表层沉积物中 MC-LR、 MC-RR、MC-YR 含量范围分别为 $0.60 \sim 26.95 、 0 \sim 0.90 、 0 \sim 8.10 \mathrm{ng} / \mathrm{g}$, 且 1 10 cm 层中 MCs 三种异构体平均含量大小顺序 为: $M C-L R>M C-Y R>M C-R R$, 其中 MC-LR、MC-RR、MC- $Y R$ 的检出率分别为 $100 \% 、 70 \% 、 92 \%$; 垂向分布上, MC-RR 含量较 低且变化不大, 而 MC-YR 和 MC-LR 含量均随沉积物深度的增加先升高后降低. 相关性分析结果表明, 表层水和混合水中 $\mathrm{MCs}$ 与总磷浓度呈显著正相关, 而与总氮浓度无显著相关性; 上覆水、间隙水以及沉积物中 MCs 与总氮、总磷浓度均呈显 著正相关.
\end{abstract}

关键词: 太湖;梅梁湾;微囊藻毒素;水体;沉积物;垂向分布

\section{Vertical distribution characteristics of microcystin concentration in water and sediment of Meiliang Bay, Lake Taihu}

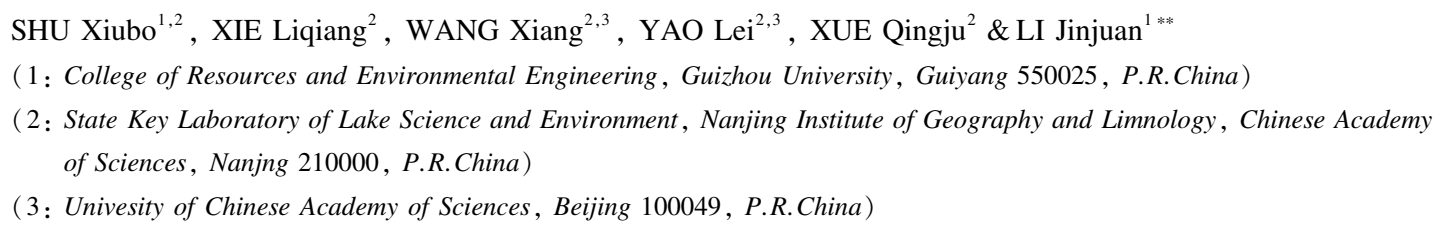

Abstract: In order to explore the vertical distribution characteristics of microcystin (MC-LR, MC-RR, MC-YR) concentration in the water body and sediment of Meiliang Bay, surface water, overlying water, mixed water, interstitial water and columnar sediment samples at six points were collected in May 2018, and the microcystin concentrations were analyzed by ultra-high performance liquid chromatography-tandem mass spectrometry( UPLC-MS/MS). The results indicated that the MC-LR, MC-RR, MC-YR concentrations in water (surface water, overlying water, mixed water and interstitial water) ranged from 11.80-1297.14, 2.50$818.40,1.80-176.00 \mathrm{ng} / \mathrm{L}$, respectively. The MC-LR concentration in surface water, overlying water and mixed water were higher than MC-RR and MC-YR concentrations, but there were no significant difference between MC-RR and MC-YR concentration. The order of MC concentrations isomer in interstitial water was: MC-LR > MC-RR > MC-YR. The MC concentrations isomer ( MC-LR, MC-RR, MC-YR) in interstitial water were all far higher than those in surface water, overlying water and mixed water in vertical

* 国家自然科学基金项目 (41877486)、国家水体污染控制与治理科技重大专项 (2018ZX07208-008，2017ZX07203004-04) 和中国科学院科技服务网络计划 (KFJ-STS-ZDTP-038-3) 联合资助. 2018-10-26 收稿; 2018-12-18 收修 改稿. 舒秀波(1994 ), 男,硕士研究生;E-mail:2635309579@ qq.com.

** 通信作者;E-mail:summy_lee@163.com. 
profiles of water, the MC isomer concentrations in surface water were slightly higher than those in overlying water. The MC isomer concentrations in mixed water were between surface water and overlying water. The MC-LR, MC-RR and MC-YR contents in sediment with a depth of $10 \mathrm{~cm}$ ranged from $0.60-26.95,0-0.90$ and $0-8.10 \mathrm{ng} / \mathrm{g}$, respectively, and the order of the average content of three MC isomers in the 1-10 cm layer was MC-LR > MC-YR > MC-RR. The detection rates of MC-LR, MC-RR and MC-YR were $100 \%, 70 \%$ and $92 \%$, respectively. In the vertical distribution of sediment, the MC-RR concentration was low and showed little change, the overall trend of MC-YR and MC-LR contents increased first and then decreased with the increasing of the sediment depth. The results of correlation analysis indicated that MC isomer concentrations in surface water and mixed water were positively correlated with total phosphorus ( TP) concentration, respectively, but did not show correlation with total nitrogen ( TN) concentration. The MC concentrations in overlying water, interstitial water and sediment were positively correlated with TN and TP concentrations, respectively.

Keywords: Lake Taihu; Meiliang Bay; microcystin; water; sediment; vertical distribution

近十年来, 太湖水体长时间处于中度富营养化状态, 蓝藻水华发生日趋频繁, 这引起了国内外学者的普 遍关注. 水华暴发期间, 微囊藻 (Microcystis)、鱼腥藻 (Anabaena) 、颤藻 (Oscillatoria) 等浮游藻类大量繁殖, 同 时藻细胞死亡破裂后会向水体释放多种蓝藻毒素, 危害水生态系统的安全 ${ }^{[1]}$, 其中微囊藻毒素 ( microcystins, 简称 MCs) 是一种分布广泛且毒性最强的蓝藻毒素. MCs 是由 7 个氨基酸组成的单环七肽, 由 于多肽组成中氨基酸种类和位置的变化, 导致了 MCs 结构上的多样性, 目前已经发现了 200 多种不同类型 的 MCs 异构体 ${ }^{[2]}$, 其中 MC-LR、MC-RR、MC-YR( $L 、 R 、 Y$ 分别代表亮氨酸、精氨酸和酪氨酸) 为最主要的 3 种 类型, 且 MC-LR 和 MC-RR 是在我国天然水体中检出频率最多和浓度最高的 MCs 异构体. MCs 有着环状和 间隔双键的结构特性, 性质稳定, 耐高温, 易溶于水 ${ }^{[3]}$, 且自然水体中 MCs 的归趋主要分为以下 5 个途径 ${ }^{[4]}$ : (1) 大量自然湖泊水体的稀释作用, (2) 沉降物或悬浮物对水体中 MCs 的吸附作用, (3) 在特定的水温及 $\mathrm{pH}$ 条件下的热分解作用, (4) 光降解作用, (5) 生物降解作用. 此外, 研究证明, MCs 还可以在鱼体、螺类及其他 水生生物体内积累 ${ }^{[5-7]}$, 一旦人类食用被 MCs 污染的水产品, MCs 很有可能在人体内富集, 从而对人体造成 很大的伤害 ${ }^{[8]}$.

目前, 国内外学者对太湖梅梁湾水体及沉积物中 MCs 浓度已开展了部分研究, 并取得了一系列的研究 结果. $\mathrm{Xu}$ 等 ${ }^{[9]}$ 用酶联免疫法 (ELISA) 分析了梅梁湾水体中 MCs 总浓度的变化, 发现一年之中的 7-10 月为 水体中 MCs 总浓度较高的时间段. Song 等 ${ }^{[10]}$ 用酶联免疫法分析了梅梁湾表层水和上覆水 MCs 总浓度的变 化, 结果表明表层水中 MCs 总浓度略高于上覆水. 高振美等 ${ }^{[11]}$ 用高效液相色谱法分析了梅梁湾水体中 MCLR、MC-RR、MC-YR 的浓度变化, 结果发现水体中 MC-LR 和 MC-RR 这 2 种异构体浓度较高. Chen 等 ${ }^{[4]}$ 用酶 联免疫法分析了梅梁湾沉积物中 MCs 总含量的变化, 研究表明蓝藻暴发期间, 沉积物中 MCs 总含量较高. 但前人的研究工作大多注重于水体, 少有对间隙水以及沉积物中 MCs 的含量报道, 没有对 MCs 的垂向分布 进行充分阐释, 且研究方法以酶联免疫法居多, 但 ELISA 是一项把抗原抗体的免疫反应和酶的高效催化作 用原理有机结合起来的一种检测技术, 其存在的问题是抗体往往只是针对某一种毒素建立起来的, 对其他 类型毒素的交叉反应性不高 ${ }^{[12]}$, 不能够较全面地反映 MCs 不同异构体在水体及沉积物中的含量分布情况.

因此本研究选取太湖蓝藻水华较严重的梅梁湾为研究区, 在蓝藻水华强度较大的 5 月 ${ }^{[13]}$, 采集梅梁湾 表层水、上覆水、混合水、间隙水以及柱状沉积物样品, 并采用超高效液相色谱一串联质谱法 (UPLC-MS/MS) 准确分析样品中 MCs 三种主要异构体 (MC-LR、MC-RR、MC-YR) 含量, 进而从垂向空间变化来详细分析蓝藻 水华期间 MCs 在梅梁湾水体以及底泥中具体分布情况, 以便后期更深人地探讨 MCs 在水体以及沉积物中 的环境归趋.

\section{1 材料与方法}

\section{1 研究区概况}

梅梁湾位于太湖的北部, 属于典型的藻型湖湾, 其水域面积约为 $100 \mathrm{~km}^{2[14]}$. 据《2017 年太湖健康状况 报告》数据显示 ${ }^{[13]}$, 梅梁湾蓝藻数量与其他湖区相比, 处于较高水平, 营养状况为中度富营养化水平, 蓝藻 水华强度较大的两个时段分别出现在 4-6 月和 8-11 月. 


\section{2 样品采集}

依据梅梁湾湖面地形、湖底淤泥深度等现状以及参考中国科学院太湖湖泊生态系统研究站布设的采样 点位, 于 2018 年 5 月在梅梁湾共布设 6 个采样点, 这 6 个点位均匀地覆盖整个梅梁湾, 具有较好的代表性 (图 1). 用 $2.5 \mathrm{~L}$ 有机玻璃采水器采集表层 $0 \sim 0.5 \mathrm{~m}$ 处水样, 取 $1 \mathrm{~L}$ 过 $\mathrm{GF} / \mathrm{C}$ 玻璃纤维滤膜 $(1.2 \mu \mathrm{m}$, Whatman, Brentford, UK), 收集滤液用于测定表层水 MCs 的浓度, 另取 $500 \mathrm{ml}$ 用于测定表层水体理化指标. 用 $2.5 \mathrm{~L}$ 有机玻璃采水器采集表层 (水下 $0 \sim 0.5 \mathrm{~m}$ ) 、中层 (水深一半处)、底层 (水底泥上 $0 \sim 0.5 \mathrm{~m}$ ) 的水样, 混 合后取 $1 \mathrm{~L}$ 过滤后用于测定混合水 MCs 浓度, 另取 $500 \mathrm{ml}$ 用于测定混合水体理化指标. 用自重力柱状采泥 器在 6 个点位采集沉积物柱状样, 每个点位采集 4 根柱子, 参考 Song 等 ${ }^{[10]}$ 对上覆水的采样方法, 在现场用 $50 \mathrm{ml}$ 针筒注射器吸取 4 根柱状泥样表层以上 $0 \sim 10 \mathrm{~cm}$ 处上覆水, 混匀后取 $1 \mathrm{~L}$ 过滤后用于测定上覆水 $\mathrm{MCs}$ 浓度, 另取 $500 \mathrm{ml}$ 用于测定上覆水体理化指标. 在每个点位选取 1 根柱子利用 $1 \mathrm{~cm}$ 切环将柱状沉积物自上 而下分割成 10 层, 混匀后装袋带回实验室, 在 $-80^{\circ} \mathrm{C}$ 条件下冷冻保存, 用于沉积物中 $\mathrm{MCs}$ 含量的测定. 剩余 6 个点位的 3 根柱子利用 $10 \mathrm{~cm}$ 切环直接切取表层 $10 \mathrm{~cm}$ 厚的沉积物, 混匀后装袋带回实验室, 在离心机 (ThermoFisher, Heraeus, Multifuge, X1R) $4^{\circ} \mathrm{C}$ 条件下以 1200 转/ $\min$ 离心 $25 \mathrm{~min}$ 得到间隙水, 经 $\mathrm{GF} / \mathrm{C}$ 滤膜 过滤后, 测定间隙水 MCs 浓度, 另取 $200 \mathrm{ml}$ 用于测定间隙水体理化指标.

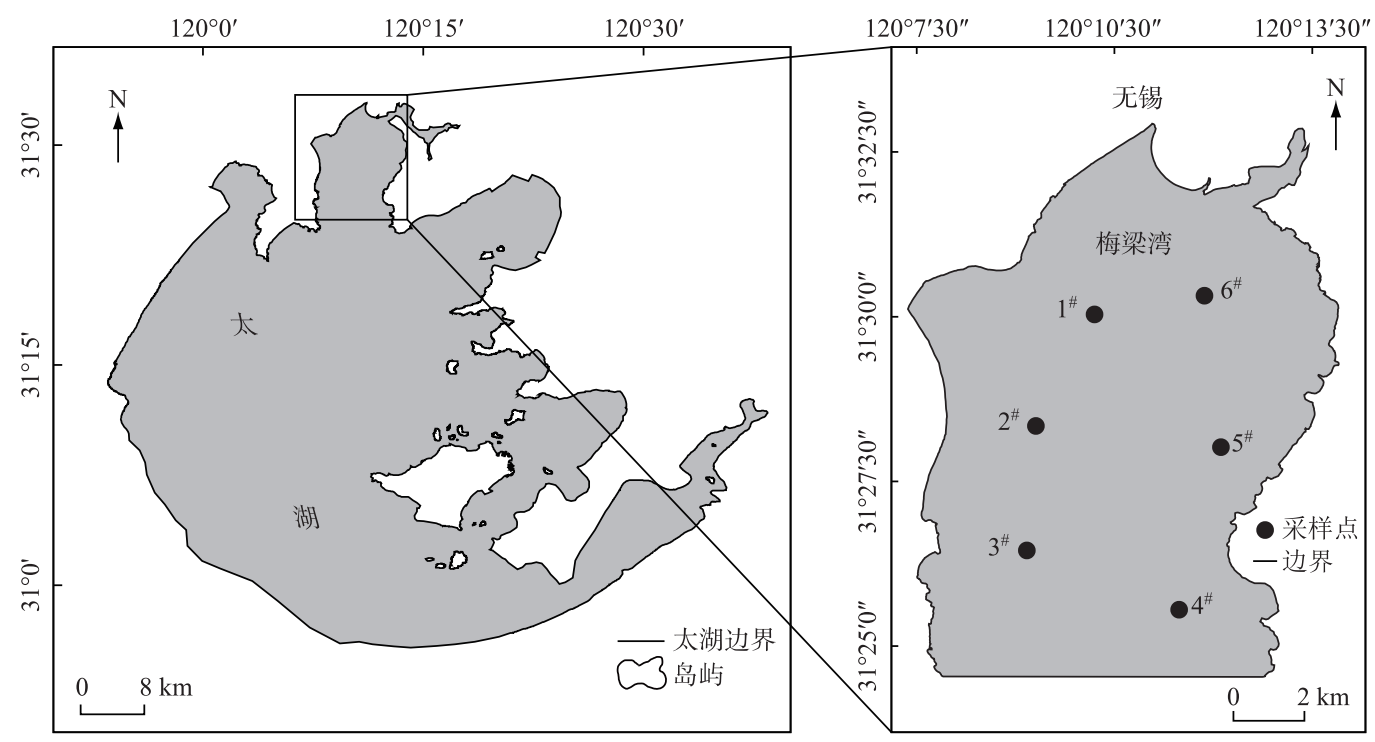

图 1 太湖梅梁湾采样点位置

Fig. 1 Distribution of sampling sites in Meiliang Bay of Lake Taihu

\section{3 样品处理与测定}

1.3.1 水体及沉积物理化指标测定 多参数水质仪 (YSI $6600 \mathrm{~V} 2$ ) 现场测定表层水、上覆水以及混合水的水 温 ( Temp)、溶解氧 (DO)、酸碱度 $(\mathrm{pH})$, 黑白透明度盘测定各点位水体透明度 $(\mathrm{SD})$; 按照《湖泊富营养化调 查规范》中的方法测定上述水体以及间隙水中总氮 $(\mathrm{TN})$ 、总磷 $(\mathrm{TP})$ 、亚硝态氮 $\left(\mathrm{NO}_{2}^{-}-\mathrm{N}\right)$ 、硝态氮 $\left(\mathrm{NO}_{3}^{-}-\mathrm{N}\right)$ 、 铵态氮 $\left(\mathrm{NH}_{4}^{+}-\mathrm{N}\right)$ 、磷酸根 $\left(\mathrm{PO}_{4}^{3-}-\mathrm{P}\right)$ 、叶绿素 $\mathrm{a}(\mathrm{Chl} . \mathrm{a})$ (间隙水除外)、高锰酸盐指数 $\left(\mathrm{COD}_{\mathrm{Mn}}\right)^{[15]}$. 沉积物中各 层样品的溶解性总氮 (STN)、溶解性总磷 (STP) 分别采用半微量凯氏定量法和硫酸一高氯酸消煮磷锄蓝比 色法测定 ${ }^{[16]}$. 梅梁湾水体及沉积物理化指标见表 1.

1.3.2 水体中溶解态 MCs 的富集 滤后的水样按 $\mathrm{Su}$ 等 ${ }^{[17]}$ 的方法处理. 将 $1 \mathrm{~L}$ 过滤后的水样以 $1 \mathrm{ml} / \mathrm{min}$ 的速 度流经活化好的 $0.2 \mathrm{~g} \mathrm{HLB}$ 富集柱 (Oasis ${ }^{\circledR}$, Waters, Milford, MA，USA), 然后将柱子用 $15 \mathrm{ml} 5 \%$ 的甲醇溶 液淋洗, 去除杂质, 最后再 $10 \mathrm{ml} 100 \%$ 的甲醇溶液洗脱, 用玻璃管收集洗脱液. 将收集的洗脱液用氮吹仪 (EYELA, MG-2200) 吹干, 残留物用 100\% 甲醇溶解, 溶解后再用真空离心浓缩仪 (EYELA, Centrifugal, 
Evaporator, CVE-2000)浓缩,最后将浓缩后的残留物 用 $500 \mu \mathrm{l} 100 \%$ 甲醇溶解, 待测.

1.3.3 沉积物中 MCs 的富集冷冻保存的样品取出, 在 $-50^{\circ} \mathrm{C}$ 条件下冷冻干燥. 在冷冻干燥完毕之后, 用 玻璃棒压散冷冻干燥后的样品, 并剔除样品中的生 物残体等杂质, 混匀并取其 $1 / 4$ 作为研磨样品, 经玛 瑙研针研细过 200 目尼龙笁后, 储存于聚乙烯瓶中 待测. 过篮后的泥样按 Chen 等 $^{[18]}$ 的方法处理. 称取 $2 \mathrm{~g}$ 泥样倒人 $50 \mathrm{ml}$ 离心管中, 用 $25 \mathrm{ml} 0.1 \mathrm{~mol} / \mathrm{L} \mathrm{ED}$ TA-0.1 mol/ $/ \mathrm{Na}_{4} \mathrm{P}_{2} \mathrm{O}_{7}$ ( $\mathrm{pH}$ 值用三氟乙酸调节为 3 ) 超声 $10 \mathrm{~min}$, 然后在 $4^{\circ} \mathrm{C} 、 10000$ 转/ min 条件下离心 $15 \mathrm{~min}$ 后得到上清液, 用 $1 \mathrm{ml}$ 移液枪吸取上清液并 转移至 $100 \mathrm{ml}$ 烧杯中, 重复上述步骤 3 次, 3 次得到 的上清液中 MCs 提取富集方法同水样.

1.3.4 MCs 的测定 用超高效液相色谱串联三重四级 杆质谱仪 (UPLC-MS/MS) 测定 MCs. 流动相为 $0.1 \%$ 甲酸 $(A)$ 和乙腈 $(B)$. 流动相梯度淋洗程序为: $0 \sim 4.0$ $\min , 75 \% \mathrm{~A} ; 4.0 \sim 4.1 \mathrm{~min}, 55 \% \mathrm{~A} ; 4.1 \sim 7.0 \mathrm{~min}, 75 \%$
表 1 梅梁湾水体及沉积物理化指标 *

Tab.1 Physical and chemical indexes in Meiliang Bay

\begin{tabular}{ccc}
\hline 理化指标 & 均值 & 范围 \\
\hline $\mathrm{Temp} /{ }^{\circ} \mathrm{C}$ & 27.50 & $27.02 \sim 28.12$ \\
$\mathrm{DO} /(\mathrm{mg} / \mathrm{L})$ & 8.28 & $5.80 \sim 9.44$ \\
$\mathrm{pH}$ & 8.85 & $8.42 \sim 9.08$ \\
$\mathrm{SD} / \mathrm{m}$ & 0.23 & $0.20 \sim 0.25$ \\
$\mathrm{TN} /(\mathrm{mg} / \mathrm{L})$ & 3.75 & $1.08 \sim 10.36$ \\
$\mathrm{TP} /(\mathrm{mg} / \mathrm{L})$ & 0.22 & $0.05 \sim 0.68$ \\
$\mathrm{NO}_{2}^{-}-\mathrm{N} /(\mathrm{mg} / \mathrm{L})$ & 0.0069 & $0.0032 \sim 0.016$ \\
$\mathrm{NO}_{3}^{-}-\mathrm{N} /(\mathrm{mg} / \mathrm{L})$ & 0.087 & $0.0018 \sim 0.18$ \\
$\mathrm{NH}_{4}^{+}-\mathrm{N} /(\mathrm{mg} / \mathrm{L})$ & 0.72 & $0.069 \sim 3.32$ \\
$\mathrm{PO}_{4}^{3-}-\mathrm{P} /(\mathrm{mg} / \mathrm{L})$ & 0.0071 & $0.0033 \sim 0.017$ \\
$\mathrm{Chl}^{-\mathrm{a}} /(\mu \mathrm{g} / \mathrm{L})$ & 51.61 & $7.49 \sim 167.89$ \\
$\mathrm{COD}_{\mathrm{Mn}} /(\mathrm{mg} / \mathrm{L})$ & 15.30 & $3.30 \sim 54.40$ \\
$\mathrm{STN} /(\mathrm{mg} / \mathrm{g})$ & 1.85 & $1.37 \sim 2.39$ \\
$\mathrm{STP} /(\mathrm{mg} / \mathrm{g})$ & 0.31 & $0.22 \sim 0.44$ \\
\hline
\end{tabular}

* Temp、DO 、pH、SD 以及 Chl.a 数据范围中未包含间隙水.

A. 流速为 $0.5 \mathrm{ml} / \mathrm{min}$, 进样量为 $10 \mu \mathrm{l}$. 色谱柱为 Acquity UPLC BEH C18 column $(100 \mathrm{~mm} \times 1 \mathrm{~mm}, 1.7 \mu \mathrm{m})$. 质谱采用正离子扫描, 多离子反应检测 $(\mathrm{MRM})$ 模式, 离子源温度 $150^{\circ} \mathrm{C}$, 喷雾电压 $5500 \mathrm{~V}$, 雾化温度 $450^{\circ} \mathrm{C}$, 雾化气压为 $55 \mathrm{kPa}$. 固体 MC-LR、MC-RR 和 MC-YR 溶于 $100 \%$ 甲醇后用于制作标准曲线(表 2), 线性范围为 $0.1 \sim 500 \mu \mathrm{g} / \mathrm{L}, \mathrm{MC}-\mathrm{LR} 、 \mathrm{MC}-\mathrm{RR} 、 \mathrm{MC}-\mathrm{YR}$ 的质荷比分别为 995.6、519.9、1045.5.

表 2 MC-LR、MC-RR、MC-YR 的标准曲线回归方程

Tab.2 Standard curve regression equations of MC-LR, MC-RR and MC-YR

\begin{tabular}{ccc}
\hline 化合物 & 标准曲线回归方程 & 相关系数 \\
\hline MC-LR & $y=7.18579 x-6.15287$ & 0.9927 \\
MC-RR & $y=26.7408 x-25.1654$ & 0.9928 \\
MC-YR & $y=4.92156 x-4.10474$ & 0.9929 \\
\hline
\end{tabular}

\section{4 统计分析}

运用 Excel 2013 和 SPSS 20.0 软件对实验数据进行统计计算、相关性分析; 此外, 采用 ArcGIS 10.3 软件 绘制太湖采样点位置图, 其余图片均由 Origin 2017 软件绘制.

\section{2 结果与分析}

\section{1 水体中 MCs 分布特征}

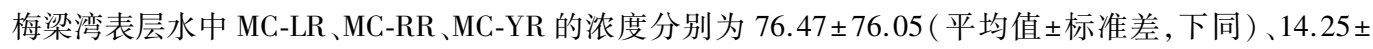
$14.52 、 6.63 \pm 3.44 \mathrm{ng} / \mathrm{L}$, 上覆水中 MC-LR、MC-RR、MC-YR 的浓度分别为 $26.63 \pm 7.97 、 3.88 \pm 1.18 、 2.94 \pm 1.31$ $\mathrm{ng} / \mathrm{L}$, 混合水中 MC-LR、MC-RR、MC-YR 的浓度分别为 $52.99 \pm 42.07 、 7.28 \pm 5.84 、 5.93 \pm 2.79 \mathrm{ng} / \mathrm{L}$, 间隙水中 MC-LR、MC-RR、MC-YR 的浓度分别为 483.88 $\pm 422.21 、 351.25 \pm 264.17 、 85.83 \pm 54.29 \mathrm{ng} / \mathrm{L}$ (图 2). 从点位分布 上看, $5^{\#}$ 点位 MCs 总浓度 (MC-LR、MC-RR、MC-YR 的浓度之和) 在表层水、上覆水、混合水以及间隙水中均 表现为最高.

各点位表层水、上覆水以及混合水中 MC-LR 的浓度均高于 MC-RR 和 MC-YR, MC-RR 和 MC-YR 之间差 别较小, 而间隙水中 MCs 三种异构体浓度大小顺序为: MC-LR $>M C-R R>M C-Y R$. 从垂直分布上看, 各点位间 隙水中 MC-LR、MC-RR、MC-YR 的浓度均高于表层水、上覆水以及混合水, 其均值分别为表层水的 $6 、 25 、 13$ 
倍, 上覆水的 $39 、 91 、 29$ 倍, 混合水的 $9 、 48 、 14$ 倍, 表层水 MCs 异构体浓度较高于上覆水, 其 MC-LR、MC-RR、 MC-YR 均值分别为表层水的 3、4、2 倍,混合水 MCs 异构体浓度介于表层水和上覆水之间.
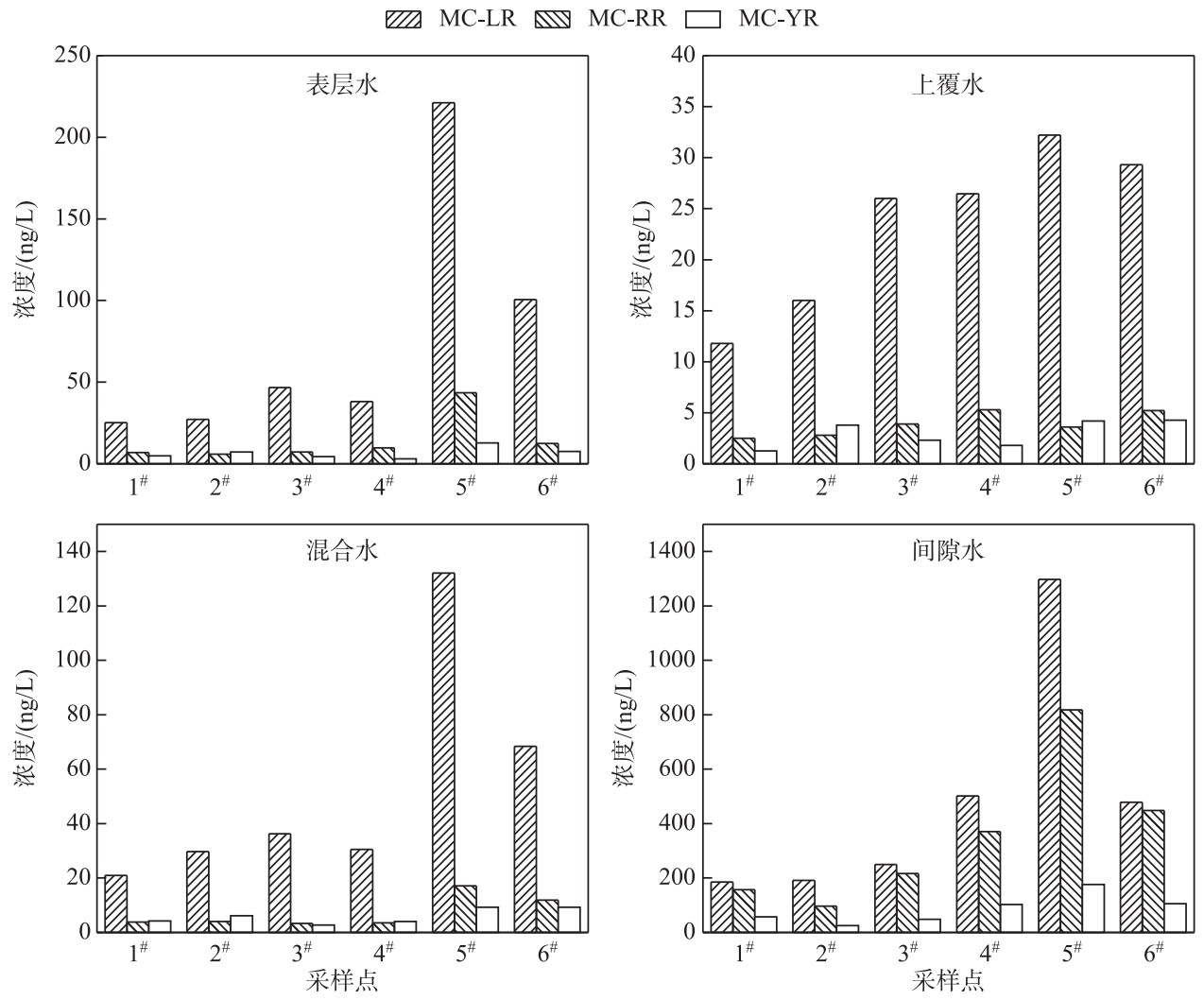

图 2 梅梁湾水体中 MCs 浓度变化

Fig. 2 The changes of MCs concentration in water of Meiliang Bay

\section{2 沉积物中 MCs 分布特征}

梅梁湾沉积物中 MC-LR 在沉积物 $1 \sim 10 \mathrm{~cm}$ 分层中均能被检测到, 且含量较高, 最大值为 $26.95 \mathrm{ng} / \mathrm{g}$, 最 小值为 $0.60 \mathrm{ng} / \mathrm{g}, \mathrm{MC}-\mathrm{RR}$ 含量较低, 含量范围为 $0 \sim 0.9 \mathrm{ng} / \mathrm{g}$, 在 6 个点位沉积物 $1 \sim 10 \mathrm{~cm}$ 分层中检出率不 高, 仅为 70\%; MC-YR 含量范围为 $0 \sim 8.10 \mathrm{ng} / \mathrm{g}$, 仅在 $1^{\#}$ 和 $3^{\#}$ 点位沉积物 $9 \sim 10 \mathrm{~cm}$ 深度处未被检测出, 检出 率为 $92 \%$, 沉积物中 MCs 三种异构体在不同深度中平均含量大小顺序为: MC-LR $>$ MC-YR $>$ MC-RR (图 3).

从垂直分布上看, MC-RR 含量较低且变化不大, 而 MC-LR 和 MC-YR 含量总体趋势为随着沉积物深度的 增加先上升后下降 ( $5^{*}$ 点位除外). 其中 $1^{\#}$ 点位和 $2^{*}$ 点位 MC-LR 和 MC-YR 含量分别在 3 和 $4 \mathrm{~cm}$ 处出现最大 值, 然后在 4 6 $6 \mathrm{~cm}$ 段急剧下降后一直处于较低状态; $3^{*}$ 点位 MC-LR 和 MC-YR 含量在 $2 \mathrm{~cm}$ 处出现最大值, 然 后在 2 5 cm 段迅速下降, 剩余 5 10 cm 段含量处于较低水平; $4^{\#}$ 点位 MC-LR 和 MC-YR 含量在 $5 \mathrm{~cm}$ 处出现最 大值, 然后在 $5 \sim 8 \mathrm{~cm}$ 段迅速下降, 之后 $8 \sim 10 \mathrm{~cm}$ 段含量处于较低水平; $5^{\#}$ 点位 MC-LR 和 MC-YR 含量在表层 $1 \sim 3 \mathrm{~cm}$ 段急剧下降之后一直处于低含量状态; $6^{*}$ 点位 MC-LR 和 MC-YR 含量具有一定波动性, MC-LR 含量在 4 和 $6 \mathrm{~cm}$ 处出现 2 个极大值, MC-YR 含量在 3 和 $6 \mathrm{~cm}$ 处出现 2 个极大值, 然后 MC-LR 和 MC-YR 含量在 $6 \mathrm{~cm}$ 处开始下降, 在 $10 \mathrm{~cm}$ 处时, MC-LR 含量降至 $4 \mathrm{ng} / \mathrm{g}, \mathrm{MC}-\mathrm{YR}$ 含量已处于较低水平(图 3).

\section{3 水体及沉积物中 MCs 相对丰度变化}

梅梁湾表层水、上覆水和混合水中 MCs 三种异构体的占比变化不大, 其中 MC-LR、MC-RR、MC-YR 的占 比范围分别为 77\% 80\% 和 11\% 15\%、7\% 10\% ; 而间隙水中 MC-LR 的占比减小为 53\%, MC-RR 的占比增 

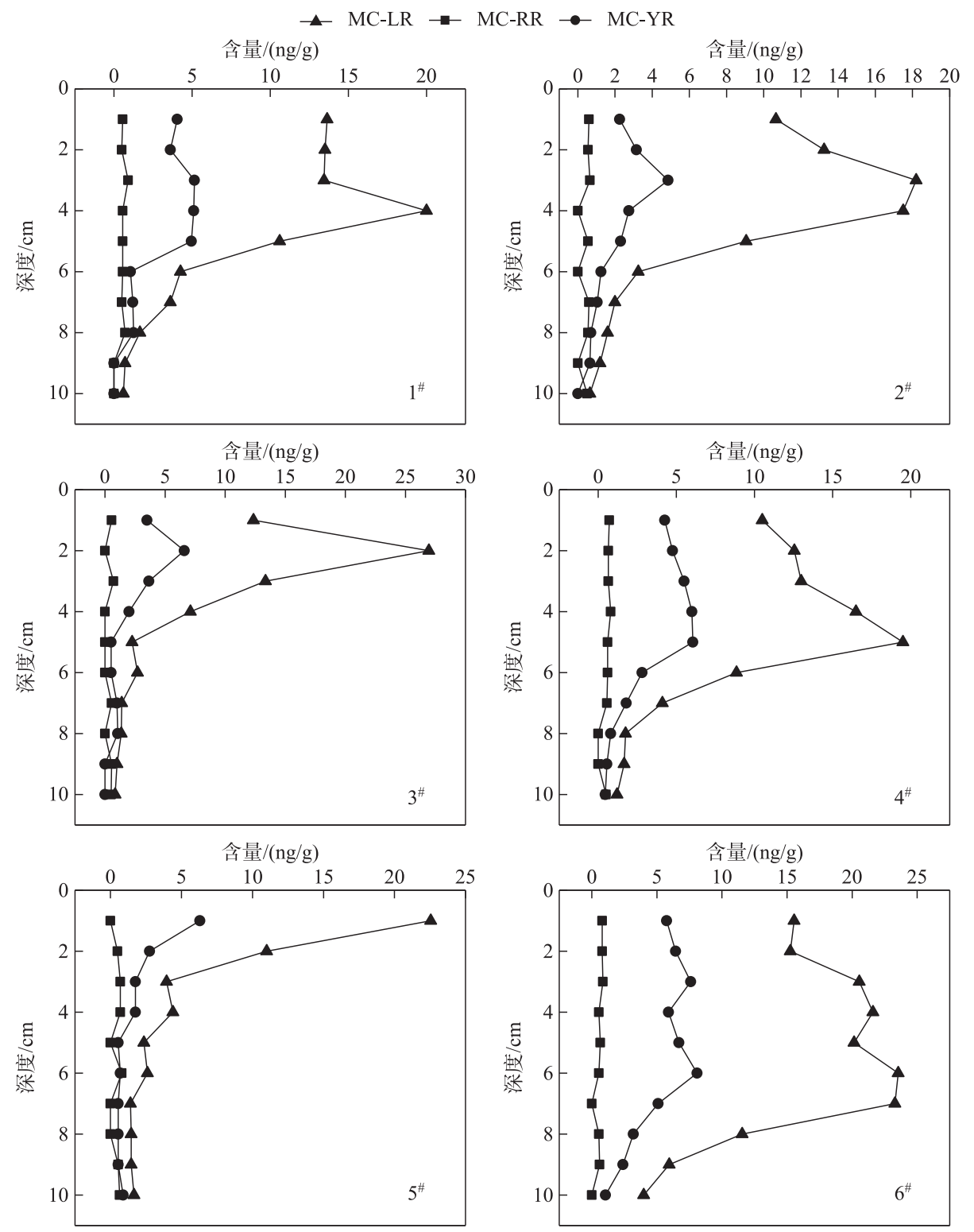

图 3 梅梁湾各样点沉积物中 $\mathrm{MCs}$ 含量垂向变化

Fig. 3 The vertical changes of MCs content in sediments of each site in Meiliang Bay

加到 38\%,MC-YR 变化不大,仅为 9\% (图 4).

梅梁湾沉积物 MC-LR 在 MCs 垂直分布中占比变化较大,最大值为 $77 \%$, 最小值为 $66 \%$, 表层 $1 \sim 2 \mathrm{~cm}$ 处 的占比 $(74.5 \%)$ 大于 9 $10 \mathrm{~cm}$ 处 $(66.5 \%)$; MC-RR 在表层 1 2 $\mathrm{cm}$ 处的占比仅为 $3 \%$, 而随着深度的增加占 比有所上升, 在 $9 \sim 10 \mathrm{~cm}$ 处增加到 $13 \%$; MC-YR 在 MCs 垂直分布中占比变化较小, 整体范围为 $18 \% \sim 26 \%$, 其中表层 1 2 cm 处的占比略高于 9 10 $\mathrm{cm}$ 处(图 5).

\section{4 水体及沉积物中 MCs 与环境因子的关系}

MCs 在水体及沉积物中的含量直接受到产毒蓝藻生物量的影响, 但也间接受到其他生物和非生物环境 因子的影响, 比如叶绿素 $\mathrm{a} 、$ 氮磷营养盐浓度等. 将水体以及沉积物中 MCs 与环境因子进行 Pearson 相关性 分析, 结果可知表层水中 MC-LR、MCs 与 TP、Chl. a、COD ${ }_{\mathrm{Mn}}$ 均呈显著正相关 $(P<0.05)$, 而与 $\mathrm{TN}$ 等其他环境因 


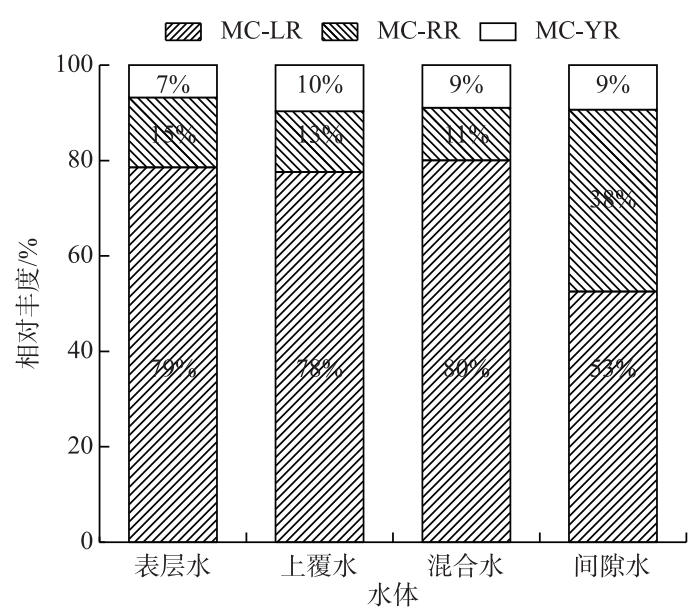

图 4 梅梁湾水体中 MCs 异构体相对丰度

Fig.4 Relative abundance of three MC congeners

( LR, RR and YR) in water of Meiliang Bay

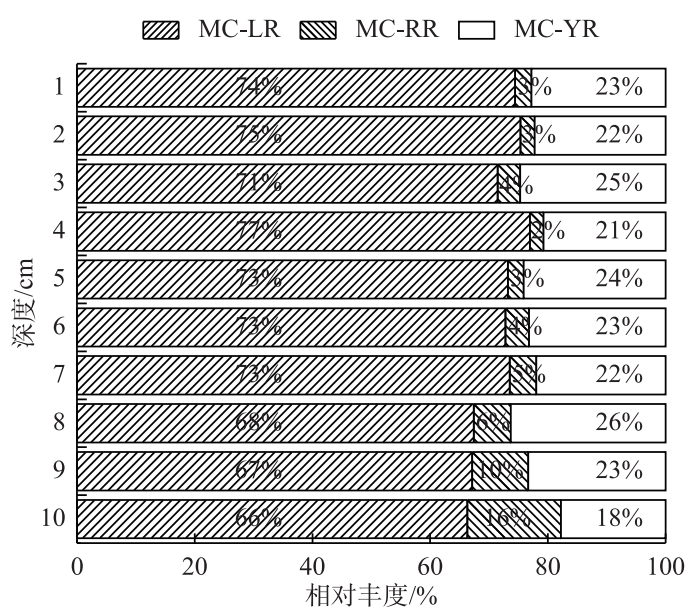

图 5 梅梁湾沉积物中 MCs 异构体相对丰度

Fig.5 Relative abundance of three MC congeners ( $\mathrm{LR}, \mathrm{RR}$ and YR) in sediment of Meiliang Bay

子均无显著相关性, MC-RR、MC-YR 与所分析的环境因子均无显著相关性; 上覆水中 MC-LR、MCs 与 TN、TP 均呈显著正相关 $(P<0.05)$, 而与 Chl.a、COD ${ }_{\mathrm{Mn}}$ 等其他环境因子均无显著相关性, 此外, MC-RR、MC-YR 与环 境因子无显著相关; 混合水中 MC-LR、MC-RR、MCs 与 $\mathrm{TP} 、 \mathrm{Chl} . \mathrm{a} 、 \mathrm{COD}_{\mathrm{Mn}}$ 均呈显著正相关 $(P<0.05)$, 而与 $\mathrm{TN}$ 等其他环境因子均无显著相关性, MC-YR 与所分析的环境因子均无显著相关; 间隙水中 MC-LR、MC-RR、 $\mathrm{MC}-\mathrm{YR} 、 \mathrm{MCs}$ 与 TN、TP 均具有显著正相关性 $(P<0.05)$, 而与 $\mathrm{COD}_{\mathrm{Mn}}$ 等其他环境因子无显著相关性 $($ 表 3$)$. 由表 4 可知, 沉积物中 MC-LR、MC-YR、MCs 含量与 STN、STP 浓度均呈显著正相关 $(P<0.01)$, 而 MC-RR 与 STN 、STP 浓度之间均无显著相关性.

\section{表 3 梅梁湾水体中 $\mathrm{MCs}$ 与环境因子的相关分析 ${ }^{1)}$}

Tab.3 The correlation between MCs and environmental factors in water of Meiliang Bay

\begin{tabular}{cccccccccc}
\hline 水体 & 类别 & $\mathrm{TN}$ & $\mathrm{NH}_{4}^{+}-\mathrm{N}$ & $\mathrm{NO}_{3}^{-}-\mathrm{N}$ & $\mathrm{NO}_{2}^{-}-\mathrm{N}$ & $\mathrm{TP}$ & $\mathrm{PO}_{4}^{3-}-\mathrm{P}$ & $\mathrm{Chl.a}$ & $\mathrm{COD}_{\mathrm{Mn}}$ \\
\hline 表层水 & $\mathrm{LR}$ & 0.680 & -0.048 & 0.373 & 0.648 & $0.891^{*}$ & -0.204 & $0.865^{*}$ & $0.897^{*}$ \\
& $\mathrm{RR}$ & 0.557 & -0.110 & 0.516 & 0.731 & 0.796 & -0.196 & 0.763 & 0.736 \\
& $\mathrm{YR}$ & 0.461 & -0.056 & 0.238 & 0.555 & 0.715 & 0.202 & 0.719 & 0.656 \\
& $\mathrm{MCs}$ & 0.658 & -0.058 & 0.393 & 0.662 & $0.876^{*}$ & -0.190 & $0.850^{*}$ & $0.837^{*}$ \\
上覆水 & $\mathrm{LR}$ & $0.935^{* *}$ & -0.237 & 0.081 & 0.176 & $0.916^{*}$ & 0.258 & 0.710 & 0.542 \\
& $\mathrm{RR}$ & 0.502 & -0.120 & -0.480 & -0.469 & 0.448 & 0.158 & 0.164 & 0.376 \\
& $\mathrm{YR}$ & 0.537 & 0.285 & -0.189 & 0.152 & 0.773 & 0.413 & 0.517 & -0.199 \\
& $\mathrm{MCs}$ & $0.915^{*}$ & -0.173 & -0.017 & 0.110 & $0.925^{* *}$ & 0.291 & 0.683 & 0.471 \\
混合水 & $\mathrm{LR}$ & 0.706 & -0.075 & 0.385 & 0.628 & $0.914^{*}$ & 0.515 & $0.872^{*}$ & $0.866^{*}$ \\
& $\mathrm{RR}$ & 0.636 & 0.144 & 0.187 & 0.536 & $0.872^{*}$ & 0.700 & $0.871^{*}$ & $0.843^{*}$ \\
& $\mathrm{YR}$ & 0.412 & 0.317 & -0.213 & 0.195 & 0.678 & 0.787 & 0.681 & 0.575 \\
& $\mathrm{MCs}$ & 0.691 & -0.029 & 0.334 & 0.602 & $0.908^{*}$ & 0.559 & $0.873^{*}$ & $0.859^{*}$ \\
间隙水 & $\mathrm{LR}$ & $0.895^{*}$ & 0.587 & 0.540 & 0.801 & $0.935^{* *}$ & 0.159 & - & 0.174 \\
& $\mathrm{RR}$ & $0.952^{* *}$ & 0.677 & 0.417 & 0.741 & $0.972^{* *}$ & 0.288 & - & 0.283 \\
& $\mathrm{YR}$ & $0.925^{* *}$ & 0.614 & 0.444 & 0.706 & $0.987^{* *}$ & 0.200 & - & 0.259 \\
& $\mathrm{MCs}$ & $0.925^{* *}$ & 0.626 & 0.493 & 0.779 & $0.959^{* *}$ & 0.210 & - & 0.221 \\
\hline
\end{tabular}

1) MCs 为 MC-LR、MC-RR、MC-YR 三者浓度之和; *表示在 0.05 水平上显著相关 (双侧); **表示在 0.01 水平上显著相 关 (双侧). 
表 4 梅梁湾沉积物中 MCs 与 STN、STP 含量的相关分析 ${ }^{1)}$

Tab.4 Correlations of STN, STP and MCs content in sediment of Meiliang Bay

\begin{tabular}{cll}
\hline & \multicolumn{1}{c}{ STN } & \multicolumn{1}{c}{ STP } \\
\hline MC-LR & $0.468^{* *}$ & $0.578^{* *}$ \\
MC-RR & 0.118 & 0.089 \\
MC-YR & $0.521^{* *}$ & $0.570^{* *}$ \\
MCs & $0.485^{* *}$ & $0.580^{* *}$ \\
\hline
\end{tabular}

1) MCs 为 MC-LR、MC-RR、MC-YR 三者浓度之和; $*$ 表示在 0.05 水平上显著相关 (双侧); $* *$ 表示在 0.01 水平上显著相 关(双侧).

\section{3 讨论}

\section{1 水体以及沉积物中 MCs 垂直分布特征分析}

本研究发现, 梅梁湾水体中 MCs 浓度在垂直空间分布上表现出一定的差异性, 表层水 MCs 异构体浓度 均略高于相应点位的上覆水浓度, 这与 Song 等 ${ }^{[10]}$ 在 2005-2006 年对梅梁湾调查得出的结果相似,但与王 经结等 ${ }^{[19]}$ 在 2009 年对太湖湖中心调查得出的结果不一致,推测可能与研究区域有关. Arnold 等 ${ }^{[20]}$ 对澳大 利亚西部某浅水湖泊研究发现, 湖面风力大小对水体中各种物质是否分层起着重要的作用. 梅梁湾与湖中 心相比, 风浪远小于湖中心, 水体易形成分层现象. 此外, 蓝藻暴发期间, 表层水中微囊藻细胞数量远高于上 覆水 ${ }^{[21]}$, 藻细胞破裂后易释放出较多的 MCs 且沉积物上覆水中微生物数量远高于表层水 ${ }^{[4]}$, 加剧上覆水中 MCs 的降解,这可能也是本研究水体中 MCs 浓度垂直空间分布差异性的另一重要原因.

微囊藻属于间歇性浮游藻类,其生命周期分为浮游生长期、下沉期、越冬期和复苏期 ${ }^{[22]}$. 水华暴发后 期, 大量的微囊藻会下沉进人沉积物, 在沉积物表面度过越冬期 ${ }^{[23-24]}$, 到了春季复苏期, 沉积物中部分微囊 藻会作为 “种源” 复苏至水柱中, 但大部分 $(65 \% \sim 85 \%)$ 微囊藻会死亡分解 ${ }^{[25]}$, 导致大量的 MCs 从藻细胞内 释放出来, 此外, 由于 MCs 为亲水性物质, 沉积物对其吸附效果较差, 只有不到 $20 \%$ 的 MCs 能够被沉积物吸 附 ${ }^{[26]}$, 因此这些释放出来的 MCs 大部分都保留在间隙水中, 成为间隙水中 MCs 异构体浓度较高的主要原 因. 本研究发现, $5^{\#}$ 点位 MCs 总浓度在各层水体中均表现为最高, 结合周边环境调查发现, $5^{\#}$ 点位近岸为居 民聚集区, 频繁的人类活动导致该区域内水体富营养化非常严重, 表层水体中 Chl. a 浓度达到了 167.89 $\mu \mathrm{g} / \mathrm{L}$, 蓝藻水华在此聚集, 腐烂分解后释放出大量的溶解态 MCs.

沉积物中 MCs 主要由沉积物中藻细胞内 MCs 以及沉积物吸附水体中溶解态 MCs 组成 ${ }^{[27]}$. 太湖 $1 \sim 15$ $\mathrm{cm}$ 段表层沉积物中微囊藻丰度会随着沉积物深度增加而先增大后减小 ${ }^{[28]}$, 这与本研究得到的沉积物中 $\mathrm{MCs}$ 含量变化趋势大体一致,而 $5^{*}$ 点位 MCs 含量变化与其他点位有所不同, 可能因为该点位经过生态疏 浚 ${ }^{[29]}$, 因此变化趋势缺少增大部分. 有研究发现 ${ }^{[30-32]}$, 沉积物 $\mathrm{pH}$ 、粒度、有机质含量以及黏土矿物含量是影 响沉积物吸附 MCs 的主要因素, 当沉积物处于低 $\mathrm{pH}$ 、细粒度时, 沉积物对 MCs 的吸附能力强. 至于有机质和 黏土矿物两者产生的影响, $\mathrm{Wu}$ 等 ${ }^{[32]}$ 研究结果表明, 当沉积物中有机质含量处于较低水平时, 黏土矿物含量 主导着对 MCs 吸附, 黏土矿物含量越高, 吸附能力越强. 结合前人对梅梁湾沉积物理化性质的调查结果 ${ }^{[33-35]}$ 可知, 梅梁湾沉积物 $\mathrm{pH}$ 、粒度均随着深度的加深而变大, 而黏土矿物含量变化与之相反, 因此沉积物吸附 MCs 的能力随着沉积物深度的加深而变弱. 但结合上述分析可知, 沉积物吸附 MCs 的量有限, 因此沉积物对 $\mathrm{MCs}$ 吸附能力的强弱的变化不会对沉积物中 MCs 含量产生趋势上的变化.

\section{2 水体以及沉积物中 MCs 相对丰度变化分析}

本研究中水体及沉积物中 MCs 以 MC-LR 为主, 这与 $\mathrm{Su}$ 等 ${ }^{[17]}$ 的研究结果大体一致, 这可能因为太湖蓝 藻细胞内合成 MC-LR 的基因种类较多, 因此藻细胞内 MCs 中 MC-LR 占比较高 ${ }^{[36]}$, 进而藻细胞死亡后释放 到湖水中的 MC-LR 较多. 此外, 自然水体中 MC-LR 的微生物降解速率较其他构型慢 ${ }^{[37-38]}$, 且微生物对 MCLR 的降解需要一段时间的滞后期 ${ }^{[39]}$. 沉积物中 MC-RR 占比较小而间隙水中 MC-RR 占比有所上升, 可能因 为在光照限制以及缺氧的环境中, 底泥中藻细胞内 MC-RR 较易释放出胞外 ${ }^{[40]}$. 此外, Chen 等 ${ }^{[41]}$ 研究发现 
MC-RR 在泥柱中迁移速率明显低于 MC-LR, 导致单位体积内 MC-RR 的含量增加, 这可能也是间隙水中 MC$\mathrm{RR}$ 占比上升的原因之一, 有关间隙水中 MCs 的报道较少, 因此间隙水中 MCs 的产生以及迁移转化机理还 需更深人的研究. MC-RR 在沉积物 9 10 cm 处占比有所增加, 主要因为 MC-LR 和 MC-YR 含量减少, 而 MC$\mathrm{RR}$ 变化不大, 而 MC-RR 变化不大的原因可能是沉积物中 MC-RR 主要存在于间隙水中, 沉积物中藻细胞内 以及被沉积物吸附的含量很低.

\section{3 水体及沉积物中 MCs 与主要环境因子的相关性探讨}

本研究中总磷浓度与各层水体和沉积物中的 MCs 总浓度均呈显著正相关, 说明总磷浓度是限制产微囊 藻毒素的藻株生长和产毒的主要因素 ${ }^{[11]}$, 总磷在蓝藻水华发生和 MCs 产生过程中发挥着重要的作用. 此外 Rinta-Kanto 等 $^{[42]}$ 和 Lee 等 ${ }^{[43]}$ 调查结果表明, 高磷有利于微囊藻产毒; 万翔等 ${ }^{[44]}$ 对洱海的研究也发现, 磷为 微囊藻产毒主要限制因子, 但磷对于微囊藻产毒的影响机制还不清楚, 这有待后续更深人的研究. 本研究发 现, 总氮浓度与表层水以及混合水中 MCs 总浓度无显著相关性, 这与高振美等 ${ }^{[11]}$ 得到的研究结果一致, 但 总氮浓度与上覆水、间隙水以及沉积物中 MCs 总含量呈显著正相关, 说明底泥中氮元素可能会影响底泥界 面处微囊藻中产毒微囊藻的相对含量 ${ }^{[45]}$, 从而进一步影响底泥界面处 MCs 的含量分布. 此外, 表层水以及 混合水中 Chl.a、COD $\mathrm{Mn}_{\mathrm{n}}$ 与 MCs 总浓度均呈显著正相关, 主要因为蓝藻水华暴发期间, 藻细胞破裂死亡后, 会 产生大量有机物及 MCs, 且 Chl.a 不会立即被降解 ${ }^{[46-47]}$, 而上覆水中 Chl.a 与 MCs 总浓度无显著相关性, 可能 因为上覆水体中 MCs 来源复杂, 会受到表层水中 MCs 的迁移或者间隙水中 MCs 的释放的影响. 本研究发 现, 水体中 MC-LR 与 TP 浓度呈显著正相关, 这与王经结等 ${ }^{[19]}$ 研究结果一致. 但袁丽娟等 ${ }^{[48]}$ 对鄱阳湖的研 究发现, 水体中 MC-LR、MC-RR、MC-YR 与 TP 浓度均呈显著负相关, 这与本研究得出的结论不一致, 主要因 为鄱阳湖存在明显的丰枯水期 ${ }^{[48]}$, 而太湖水位变化相对较小 ${ }^{[49]}$, 鄱阳湖藻类生长旺盛的季节正好处于丰水 期, TP 浓度被大量水体稀释,所以与 MCs 含量呈负相关.

\section{4 结论}

1) 从垂直分布上看, 梅梁湾间隙水中 MCs 异构体 (MC-LR、MC-RR、MC-YR) 浓度均远高于表层水、上覆 水以及混合水, 表层水 MCs 异构体浓度较高于上覆水, 混合水 MCs 异构体浓度介于表层水和上覆水之间; 沉积物中 MC-RR 含量较低且变化不大,而 MC-YR 和 MC-LR 含量均随沉积物深度的增加先上升后下降.

2) 梅梁湾表层水、上覆水以及混合水中 MC-LR 为主要的毒素类型, 占比约为 79\% ; 而间隙水中 MC-LR 的占比下降至 53\%, MC-RR 的占比上升到 38\%, MC-YR 变化不大, 维持在 $9 \%$; 在表层 $10 \mathrm{~cm}$ 沉积物中 $\mathrm{MC}-$ LR 为主要的毒素类型, 且随着沉积物深度的增加, MC-LR 的占比小幅度下降, 而 MC-RR 的占比有所增加, MC-YR 的占比变化不大.

$3)$ 相关性分析表明, 表层水和混合水中 MCs 与 TP 浓度呈显著正相关 $(P<0.05)$, 而与 TN 浓度无显著相 关性; 上覆水、间隙水以及沉积物中 MCs 与 TN 、 TP 浓度均呈显著正相关 $(P<0.05)$.

\section{5 参考文献}

[ 1 ] Falconer IR. Tumor promotion and liver injury caused by oral consumption of cyanobacteria. Environ Toxic Water, 1991,6 (2) : 177-184. DOI: $10.1002 /$ tox.2530060207.

[ 2 ] Meriluoto J, Spoof L, Codd GA eds. Handbook of cyanobacterial monitoring and cyanotoxin analysis. Chichester: John Wiley \& Sons, 2017: 526-537. DOI: 10.1002/9781119068761.

[ 3 ] Dziga D, Wasylewski M, Wladyka B et al. Microbial degradation of microcystins. Chem Res Toxicol, 2013, 26(6): 841852. DOI: $10.1021 / \mathrm{tx} 4000045$.

[ 4 ] Chen W, Song L, Peng L et al. Reduction in microcystin concentrations in large and shallow lakes: Water and sedimentinterface contributions. Water Research, 2008, 42(3) : 763-773. DOI: 10.1016/j.watres.2007.08.007.

[ 5 ] Jia J, Luo W, Lu Y et al. Bioaccumulation of microcystins (MCs) in four fish species from Lake Taihu, China: Assessment of risks to humans. Science of the Total Environment, 2014, 487 (1) : 224-234. DOI: 10.1016/j. scitotenv. 2014. 04.037 .

[ 6 ] Zhang J, Wang Z, Song Z et al. Bioaccumulation of microcystins in two freshwater gastropods from a cyanobacteria-bloom 
plateau lake, Lake Dianchi. Environmental Pollution, 2012, 164(1) : 227-234. DOI: 10.1016/j.envpol.2012.01.021.

[ 7 ] Zhang D, Xie P, Chen J et al. Determination of microcystin-LR and its metabolites in snail (Bellamya aeruginosa), shrimp (Macrobrachium nipponensis) and silver carp (Hypophthalmichthys molitrix) from Lake Taihu, China. Chemosphere, 2009, 76(7) : 974-981. DOI: 10.1016/j.chemosphere.2009.04.034.

[ 8 ] Zhang D, Xie P, Liu Y et al. Transfer, distribution and bioaccumulation of microcystins in the aquatic food web in Lake Taihu, China, with potential risks to human health. Science of the Total Environment, 2009, 407(7) : 2191-2199. DOI: 10.1016/j.scitotenv.2008.12.039.

[ 9 ] Xu Q, Chen W, Gao G. Seasonal variations in microcystin concentrations in Lake Taihu, China. Environ Monit Assess, 2008, 145(1-3) : 75-79. DOI: 10.1007/s10661-007-0016-5

[10] Song L, Chen W, Peng L et al. Distribution and bioaccumulation of microcystins in water columns: A systematic investigation into the environmental fate and the risks associated with microcystins in Meiliang Bay, Lake Taihu. Water Research, 2007, 41(3) : 2853-2864. DOI: 10.1016/j.watres.2007.02.013.

[11] Gao ZM, Zhao ZH, Zhang B et al. Seasonal variation of microcystins concentration and influencing factors in Meiliang Bay, Lake Taihu. Ecology and Environmental Sciences, 2011, 20(6/7): 1063-1067. [高振美, 赵中华, 张波等. 太湖 梅梁湾水体微囊藻毒素含量的季节变化特征及其影响因素研究. 生态环境学报, 2011, 20(6/7): 1063-1067.]

[12] Wang L, Li XY, Xue WT et al. Review on research progress of microcystins detection. Food Science, 2005, 26(Z) : 136138. [王蕾, 李小艳, 薛文通等. 微囊藻毒素检测方法研究进展. 食品科学, 2005, 26 (Z) : 136-138. ]

[13] Water resources department of the Taihu basin Jiangsu provincial department of water resources, Zhejiang provincial department of water resources, Shanghai municipal water bureau eds. Taihu health report. 2017. [水利部太湖流域管理局, 江 苏省水利厅，浙江省水利厅，上海市水务局. 太湖健康状况报告. 2017.]

[14] Ke ZX, Xie P, Guo LG et al. Effects of large bio-manipulation fish pen on community structure of crustacean zooplankton in Meiliang Bay of Taihu Lake. Chinese Journal of Applied Ecology, 2012, 23(8) : 2270-2276. DOI: 10.13287/j.10019332.2012.0317. [柯志新, 谢平, 过龙根等. 太湖梅梁湾大型控藻围栏对浮游甲壳动物群落结构的影响. 应用生态 学报, 2012, 23(8): 2270-2276.]

[15] Jing XC, Tu QY eds. The standard methods in lake eutrophication investigation: The second edition. Beijing: China Environmental Science Press, 1990: 229-230. [ 金相灿, 屠清瑛. 湖泊富营养化调查规范:第 2 版. 北京: 中国环境科学 出版社, 1990: 229-230.]

[16] Bao SD ed. Agricultural soil analysis: The third edition. Beijing: China Agricultural Press, 1981: 30-33. [鲍土旦. 土壤 农化分析:第 3 版. 北京: 中国农业出版社, 1981: 30-33.]

[17] Su X, Steinman AD, Xue Q et al. Evaluating the contamination of microcystins in Lake Taihu, China: The application of equivalent total MC-LR concentration. Ecological Indicators, 2018, 89: 445-454. DOI: 10.1016/j.ecolind.2017.11.042.

[18] Chen W, Li L, Gan N et al. Optimization of an effective extraction procedure for the analysis of microcystins in soils and lake sediments. Environmental Pollution, 2006, 143(2) : 241-246. DOI: 10.1016/j.envpol.2005.11.030.

[19] Wang JJ, Yang J, Xian QM et al. Characteristic of microcystin distributions and its relationships with environmental factors in Lake Taihu. J Lake Sci, 2011, 23(4) : 513-519. DOI: 10.18307/2011.0404. [王经结, 杨佳, 鲜啟鸣等. 太湖微囊 藻毒素时空分布特征及与环境因子的关系. 湖泊科学, 2011, 23(4) : 513-519.]

[20] Arnold TN, Oldham CE. Trace-element contamination of a shallow wetland in Western Australia. Marine \& Freshwater Research, 1997, 48(6) : 531-539. DOI: 10.1071/MF96088.

[21] Zhu W, Li M, Dai X et al. Differences in vertical distribution of Microcystis morphospecies composition in a shallow hypertrophic lake (Lake Taihu, China). Environmental Earth Sciences, 2015, 73(9) : 1-10. DOI: 10.1007/s12665-0143826-0.

[22] Ihle T, Jähnichen S, Benndorf J. Wax and wane of Microcystis (Cyanophyceae) and microcystins in lake sediments: A case study in Quitzdorf Reservoir (Germany). Journal of Phycology, 2010, 41 (3) : 479-488. DOI: 10.1111/j. 15298817.2005.00071.x.

[23] Boström B, Pettersson AK, Ahlgren I. Seasonal dynamics of a cyanobacteria-dominated microbial community in surface sediments of a shallow, eutrophic lake. Aquatic Sciences, 1989, 51(2) : 153-178. DOI: 10.1007/BF00879300.

[24] Tsujimura S, Tsukada H, Nakahara H et al. Seasonal variations of Microcystis populations in sediments of Lake Biwa, Japan. Hydrobiologia, 2000, 434(1/2/3) : 183-192. DOI: 10.1023/A:1004077225916. 
[25] Wang CB. Study on the dynamic and affecting factors of Microcystis and microcystin in sediment[Dissertation]. Beijing: University of Chinese Academy of Sciences, 2014. [王纯波. 微囊藻及其毒素在沉积物中的动态变化及影响因素研究 [学位论文]. 北京: 中国科学院大学, 2014.]

[26] Lahti K, Rapala J, Färdig M et al. Persistence of cyanobacterial hepatotoxin, microcystin-LR in particulatematerial and dissolved in lake water. Water Research, 1997, 31(5) : 1005-1012. DOI: 10.1016/S0043-1354(96)00353-3.

[27] Song H, Coggins LX, Reichwaldt ES et al. The importance of lake sediments as a pathway for Microcystin dynamics in shallow eutrophic lakes. Toxins, 2015, 7(3) : 900-918. DOI: 10.3390/toxins7030900.

[28] Ye W, Tan J, Liu X et al. Temporal variability of cyanobacterial populations in the water and sediment samples of Lake Taihu as determined by DGGE and real-time PCR. Harmful Algae, 2011, 10( 5 ) : 472-479. DOI: 10.1016/j.hal.2011. 03.002 .

[29] Ma QQ, Wang B, Zhang ZY et al. Study on heavy metals pollution in sediments from North of Taihu Lake and its biological toxicity. Asian Journal of Ecotoxicology, 2016, 11(3) : 204-210. DOI: 10.7524/AJE.1673-5897.20150901001. [马青 清, 王博, 张责研等. 太湖北部表层沉积物重金属污染及其生物毒性研究. 生态毒理学报, 2016, 11 (3): 204-210.]

[30 ] Miller MJ, Critchley MM, Hutson J et al. The adsorption of cyanobacterial hepatotoxins from water onto soil during batch experiments. Water Research, 2001, 35(6) : 1461-1468. DOI: 10.1016/S0043-1354(00) 00419-X.

[31] Grützmacher G, Wessel G, Klitzke S et al. Microcystin elimination during sediment contact. Environmental Science \& Technology, 2010, 44(2) : 657-662. DOI: 10.1021/es9016816.

[32] Wu X, Xiao B, Li R et al. Mechanisms and factors affecting sorption of microcystins onto natural sediments. Environmental Science \& Technology, 2011, 45(7) : 2641-2647. DOI: 10.1021/es103729m.

[33] Lu M, Zhang WG, Shi YX et al. Vertical variations of metals and nutrients in sediments from northern Taihu Lake and the influencing factors. J Lake Sci, 2003, 15(3): 213-220. DOI: 10.18307/2003.0304. [陆敏, 张卫国, 师育新等. 太湖 北部沉积物金属和营养元素的垂向变化及其影响因素. 湖泊科学, 2003, 15(3): 213-220.]

[34] Zhao XQ, Yang LY, Yu ZY et al. Temporal and spatial distribution of physicochemical characteristics and nutrients in sediments of Lake Taihu. J Lake Sci, 2007, 19(6): 698-704. DOI: 10.18307/2007.0612. [赵兴青, 杨柳燕, 于振洋等. 太湖沉积物物理化性质及营养盐的时空变化. 湖泊科学, 2007, 19(6): 698-704.]

[35] Wang FW. Temporal and spatial variations of physico-chemical characteristics in sediments of Lake Taihu, China[Dissertation]. Guangzhou: Jinan University, 2011. [王锋文. 太湖沉积物物理化学性质时空变化特征研究 [学位论文]. 广 州: 暨南大学, 2011.]

[36] Su X, Xue Q, Steinman AD et al. Spatiotemporal dynamics of microcystin variants and relationships with environmental parameters in Lake Taihu, China. Toxins, 2015, 7(8) : 3224-3244. DOI: 10.3390/toxins7083224.

[37] Park HD, Sasaki Y, Maruyama T et al. Degradation of the cyanobacterial hepatotoxin microcystin by a new bacterium isolated from a hypertrophic lake. Environmental Toxicology, 2010, 16(4) : 337-343. DOI: 10.1002/tox.1041.

[38] Yan H, Deng YM, Zou H et al. Isolation and activity of bacteria for the biodegradation of microcystins. Environmental Science, 2004, 25(6) : 336-339. DOI: 10.13227/j.hjkx.2004.06.010. [间海, 邓义敏, 邹华等. 降解微囊藻毒素菌种的 笁选和活性研究. 环境科学, 2004, 25(6): 336-339.]

[39] Jones GJ, Orr PT. Release and degradation of microcystin following algaecide treatment of a Microcystis aeruginosa bloom in a recreational lake, as determined by HPLC and protein phosphatase inhibition assay. Water Research, 1994, 28(4) : 871876. DOI: $10.1016 / 0043-1354$ ( 94 ) 90093-0.

[40] Van DB, Verspagen JM, Lürling M et al. The ecological stoichiometry of toxins produced by harmful cyanobacteria: an experimental test of the carbon-nutrient balance hypothesis. Ecology Letters, 2010, 12(12): 1326-1335. DOI: 10.1111/j. 1461-0248.2009.01383.x.

[41] Chen W, Song L, Gan N et al. Sorption, degradation and mobility of microcystins in Chinese agriculture soils: Risk assessment for groundwater protection. Environmental Pollution, 2006, 144 (3): 752-758. DOI: 10.1016/j. envpol. 2006. 02.023 .

[42] Rinta-Kanto JM, Konopko EA, Debruyn JM et al. Lake Erie Microcystis: Relationship between microcystin production, dynamics of genotypes and environmental parameters in a large lake. Harmful Algae, 2009, 8(5) : 665-673. DOI: 10.1016/ j.hal.2008.12.004. 
[43] Lee TA, Rollwagen-Bollens G, Bollens SM et al. Environmental influence on cyanobacteria abundance and microcystin toxin production in a shallow temperate lake. Ecotoxicology \& Environmental Safety, 2015, 114: 318-325. DOI: 10.1016/j. ecoenv.2014.05.004.

[44] Wan X, Tai YP, Wang R et al. Distribution patterns of microcystins-producing Microcystis and microcystin-LR during blooms in drinking watersource areas of Lake Erhai. Acta Scientiae Circumstantiae, 2017, 37 (6) : 2040-2047. DOI: 10. 13671/j.hjkxxb.2017.0037. [万翔, 邰义萍, 王瑞等. 洱海水华期间饮用水源区产毒微囊藻和微囊藻毒素-LR 的分 布特征. 环境科学学报, 2017, 37(6): 2040-2047.]

[45] Wu WX. Unique spatiotemporal distribution of total and toxic Microcystis populations in water and sediments in Lake Taihu [Dissertation]. Shanghai : East China University of Science and Technology, 2016. [ 吴文仙. 太湖水体和底泥中总微囊 藻与产毒微囊藻丰度的分布特征 [学位论文]. 上海: 华东理工大学, 2016.]

[46] Zhang YL, Yang LY, Qing BQ et al. Spatial distribution of COD and the correlations with other parameters in the northern region of Lake Taihu. Environmental Science, 2008, 29(6) : 1457-1462. DOI: 10.13671/j.hjkxxb.2017.0037. [张运林, 杨龙元, 秦伯强等. 太湖北部湖区 COD 浓度空间分布及与其他要素的相关性研究. 环境科学, 2008, 29(6): 1457-1462.]

[47] Wang JG, Zou H, Zhang Q et al. Spatial and temporal distribution of microcystin in Taihu Lake. Research of Environmental Sciences, 2014, 27(7) : 696-703. [王靖国, 邹华, 张强等. 太湖微囊藻毒素的时空分布特征. 环境科学研究, $2014,27(7): 696-703$.

[48] Yuan LJ, Liao QG, Zhang L et al. Seasonal and spatial variations of microcystins and their relationships with physiochemical and biological factors in Poyang Lake. Environmental Science, 2018, 39(1): 450-459. DOI: 10. 13227/j. hjkx. 201708227. [袁丽娟, 廖且根, 张莉等. 鄱阳湖微囊藻毒素时空分布格局及其与理化和生物因子的关系. 环境科 学, 2018, 39(1): 450-459.]

[49] Wang LZ, Hu QF, Hu Y et al. Changes and cause analysis of water level characteristic factors in Taihu Lake during period from 1954 to 2013. Journal of Hohai University: Natural Sciences, 2016, 44(1): 14-19. [王否之, 胡庆芳, 胡艳等. 1954-2013 年太湖水位特征要素变化及成因分析. 河海大学学报: 自然科学版, 2016, 44(1): 14-19.] 